\title{
L'EUVRE LITTÉRAIRE AU CENTRE DE LA MÉDIATION LINGUISTIQUE AU NIVEAU UNIVERSITAIRE : REGARDS CROISÉS
}

\author{
UDC 811.133.1'243:378.147
}

\section{Ljiljana Đurić}

\author{
Université de Belgrade, Faculté des lettres, Belgrade, Serbie
}

\begin{abstract}
Résumé. Le Cadre européen commun de référence pour les langues introduisait en 2001 la notion de médiation linguistique, donnant une forte impulsion à la transformation de la traditionnelle activité de traduction en classe de langue étrangère (qui, au niveau universitaire, prenait surtout la forme de traduction de textes littéraires), transformation allant dans le sens de diverses formes de reformulation de textes, le plus souvent non littéraires. Cet article présente les résultats d'une recherche interprétative contextualisée dont l'objectif était de vérifier, à partir d'une activité de médiation interlangues écrite (du serbe en français) en contexte pédagogique, si la compétence linguistique des étudiants de deuxième année de langue et de littérature françaises, jugée au niveau B1-B2 pour la plupart, leur permettait d'interpréter, d'analyser et d'évaluer en français une cuvre littéraire complexe, telle que Le Roman de Londres de Miloš Crnjanski. La qualité des productions des étudiants est mise en relation avec, d'une part, les descripteurs proposés dans le Volume complémentaire avec de nouveaux descripteurs, publié en 2018 par le Conseil de l'Europe, qui mettent le texte littéraire au cour de l'activité langagière de médiation, et, d'autre part, avec le degré de satisfaction du travail accompli des étudiants eux-mêmes. Des informations sur les stratégies de planification sont également révélées.
\end{abstract}

Mots-clés : enseignement universitaire du français en Serbie, médiation écrite L1>L2, texte littéraire, réaction personnelle, stratégies de planification, culture éducative

\section{CONTEXTE DE LA RECHERCHE}

Si l'approche communicative a banni la traduction de la classe de langue étrangère dans l'éducation formelle pré-universitaire - qui cherchait par là à se rapprocher de l'éducation non formelle, réputée plus efficace dans l'enseignement des langues vivantes -, tel n'a pas été le cas au niveau universitaire : en Serbie, par exemple, à la Faculté des Lettres de l'Université

Submitted July 2, 2019; Accepted September 9, 2019

Corresponding author: Ljiljana Đurić

University of Belgrade, Faculty of Philology

E-mail: 1jiljana.djuric.55@gmail.com 
de Belgrade, la traduction - thème et version - n'a jamais cessé d'être enseignée dans des cours dirigés spécialement prévus à cet effet ${ }^{1}$, quelle que soit la langue étudiée.

Pour ce qui est du Département des langues romanes de la Faculté des Lettres de l'Université de Belgrade, et plus particulièrement des études de langue et de littérature françaises, la matière chargée de dispenser cet enseignement et d'évaluer les compétences des étudiants, matière à laquelle nous nous référerons tout au long de cet article comme étant le cadre dans lequel nous avons réalisé notre recherche, est Le français contemporain ${ }^{2}$.

Deux impulsions, que nous avons déjà évoquées plus en détail dans un autre article la publication du Cadre européen commun de référence pour les langues ${ }^{3}$ en 2001 qui introduisait la notion de médiation linguistique, et les réformes universitaires enclenchées par le processus de Bologne (Đurić 2016, 438, 439) - ont permis au Département d'introduire, à côté des exercices de thème et de version (traduction de textes littéraires), d'autres formes de médiation de textes généralement non littéraires, à l'exception de celle au programme du deuxième semestre en deuxième année : résumés et compte rendus, synthèses, développements explicatifs, etc. Ces activités de médiation écrite interlangues (du serbe vers le français) ont pour objectif communicatif de former les étudiants à :

- (première année, premier semestre) présenter par écrit en français un personnage de la culture serbe en partant d'un texte non littéraire en serbe (développement explicatif) ;

- (première année, deuxième semestre) présenter par écrit en français un lieu en Serbie en partant de textes non littéraires en serbe (synthèse) ;

- (deuxième année, premier semestre) présenter par écrit en français un événement culturel ayant (eu) lieu en Serbie en partant de textes non littéraires en serbe (synthèse) ;

- (deuxième année, deuxième semestre) présenter par écrit en français son avis et ses réactions concernant une œuvre littéraire écrite en langue serbe, Le Roman de Londres de Miloš Crnjanski ${ }^{4}$ (essai critique).

La recherche exposée dans cet article se place dans le contexte de cette dernière activité, que nous réalisons avec les étudiants depuis plus de dix ans. Jusqu'à récemment cependant, nous ne disposions pas de descripteurs appropriés permettant d'établir une relation objective entre la complexité de la tâche et le niveau de compétence de communication des étudiants jugée globalement entre les niveaux B1 et B2 pour la plupart, compétence qu'on peut appeler

\footnotetext{
${ }^{1}$ C'est le cas en Serbie, mais également en France. D. Weissmann fait un commentaire succinct de la situation (Weissmann $2012: 314-315$ ).

${ }^{2}$ Et plus particulièrement Le français contemporain IV (deuxième semestre de la deuxième année d'études de licence). L'examen est constitué d'une épreuve écrite et d'une épreuve orale, l'épreuve écrite prévoyant un exercice de version, un exercice de thème et une dictée.

${ }^{3}$ Dorénavant CECR 2001.

${ }^{4}$ L'écrivain Miloš Crnjanski (1893-1977) a d'abord été poète et dramaturge, puis journaliste, professeur et diplomate. Après la Deuxième Guerre mondiale, il a passé de longues années en exil à Londres. Revenu à Belgrade en 1965, il y écrit en 1971 Le Roman de Londres qui connait immédiatement un grand succès et obtient un prix littéraire important, le Prix de NIN. Voici ce qu'en dit Milivoj Srebro dans son article consacré à l'écrivain et publié dans la revue Serbica: «Enfin, une place à part dans l'opus de Crnjanski - celle que l'on réserve aux livres testamentaires - appartient au Roman de Londres [Roman o Londonu, 1971]. Dans ce « chant du cygne » qui devait le libérer définitivement des traumatismes de l'exil, l'écrivain revient aux thèmes de l'errance et du déracinement : il se penche, cette fois, sur le sort des émigrés russes, proscrits et déshérités, qui se sont retrouvés, au lendemain de la Seconde Guerre mondiale, à Londres, à la merci d'une impitoyable Babylone moderne. Précisément, ce roman est construit autour de l'histoire d'un couple, le prince Repnine et sa femme Nadia, pour se transformer en une épopée poignante sur l'amour et le sacrifice, le destin et le « hasard comédien », bref, en une réflexion sur la condition humaine qui peut être lue à la fois comme le miroir de l'odyssée personnelle de l'auteur et comme une allégorie sur l'errance d'Adam et Eve, chassés à jamais de leur Ithaque. »
} 
ici compétence de médiation. En proposant de nouvelles échelles - Exprimer une réaction personnelle à l'égard des textes créatifs et Analyser et critiquer des textes créatifs - le Volume complémentaire avec de nouveaux descripteurs, publié en 2018 par le Conseil de l'Europe, a mis le texte littéraire au cœur de l'activité langagière de médiation et nous a permis d'avoir un appui solide pour analyser la complexité de la tâche et juger, en fin de compte, si elle est ou non adaptée au niveau des étudiants, c'est-à-dire s'ils peuvent en tirer profit aussi bien pour leur apprentissage de la langue française que pour l'amélioration de leurs stratégies de médiation en vue de leur future profession.

\section{CADRE THÉORIQUE DE LA RECHERCHE : LA PERSPECTIVE ACTIONNELLE}

Pour cette recherche en didactique du français langue étrangère, qui, par certains aspects, peut être vue comme une recherche-action (elle a été conçue, en effet, dans le cadre du paradigme critique et a prévu, sur le plan méthodologique, le cycle planification action - observation - réflexion ${ }^{5}$ ), nous adoptons l'un des principaux postulats du CECR, à savoir que l'apprenant-utilisateur d'une langue est un acteur social qui réalise des tâches : «La perspective privilégiée ici est, très généralement aussi, de type actionnel en ce qu'elle considère avant tout l'usager et l'apprenant d'une langue comme des acteurs sociaux ayant à accomplir des tâches (qui ne sont pas seulement langagières) dans des circonstances et un environnement donné, à l'intérieur d'un domaine d'action particulier. » (CECR 2001, 15). Nous voyons également nos apprenants comme de futurs professionnels dans le domaine général des langues et des cultures et partons de l'hypothèse que, quel que soit plus tard leur domaine de spécialité (traduction, enseignement, recherche, etc.) et le secteur dans lequel ils travailleront un jour (métiers du livre, média, tourisme, etc.), ils auront besoin de mettre en œuvre leur capacité à s'exprimer en langue étrangère à propos de leur propre culture. Quant à l'environnement social dans lequel nos apprenants agiront en langue-culture étrangère - ou la «nouvelle situation sociale de référence », pour reprendre le terme de Christian Puren (Puren 2012, 9) - nous la voyons comme une société multilingue et multiculturelle dans une Europe multilingue et multiculturelle dans laquelle ils « devront vivre et travailler en permanence avec des personnes de langues et de cultures entièrement ou partiellement différentes » (Puren 2012, 9).

Un autre postulat tient une place importante dans le cadre théorique de notre recherche, celui que «toute situation d'enseignement est culturellement marquée » (Beacco 2008, 6). En ce qui concerne le travail sur le texte littéraire, la culture éducative serbe diffère sensiblement de la culture éducative française : en Serbie, comme dans beaucoup d'autres pays en réalité, on ne pratique pas l'explication de texte telle qu'elle est traditionnellement pratiquée dans les établissements scolaires français, ni d'ailleurs le très codifié exercice de la dissertation littéraire. Nos attentes par rapport au contenu des productions des étudiants tenaient compte, dans cette activité d'évaluation formative, du manque d'entrainement et de familiarité évoqués, et notre grille d'évaluation ne comprenait pas de critères tels que Capacité d'amorcer / d'amener / de problématiser le sujet ou bien, Structure du texte / Plan répondant à la problématique, etc., toujours présents dans les grilles d'évaluation françaises, en partie même dans celles du DELF et du DALF.

\footnotetext{
${ }^{5}$ L'explication de ce cycle au sein de l'épistémologie didactique et critique de la recherché-action est donnée de façon approfondie dans Pešić i saradnici (1998, 19-31).
} 


\section{CHOIX MÉTHOdOLOGIQUES ET SCÉNARIO DE L’ACTIVITÉ ET DE LA RECHERCHE}

Notre recherche interprétative contextualisée, dont l'objectif premier est donc de vérifier, à partir d'une activité de médiation interlangues écrite (du serbe en français) en contexte pédagogique, si la compétence linguistique des étudiants de deuxième année de langue et de littérature françaises (jugée au niveau B1-B2 pour la plupart, rappelons-le) leur permettait d'analyser, d'évaluer et surtout d'interpréter en français une œuvre littéraire complexe, telle que Le Roman de Londres de Miloš Crnjanski, croise la perspective de l'enseignantévaluateur-chercheur, comme nous nous définissons, et celle des étudiants eux-mêmes par l'observation de leurs productions écrites, ce corpus de productions écrites en français ayant été complété de réponses que les étudiants ont données en serbe (et dans la langue maternelle de l'étudiant étranger) lors du cours qui a suivi l'activité sur Le Roman de Londres.

A/ décembre 2018 : les étudiants sont invités à lire Le Roman de Londres dans son intégralité (après vérification, il est constaté que personne ne l'avait lu précédemment; cependant, les étudiants connaissent l'auteur dont ils ont lu au lycée le roman Les Migrations - lecture obligatoire) ; au cours du même mois, un rappel est donné ; l'enseignant précise que sera demandée uniquement la réaction personnelle de l'étudiant et non le compte rendu de ce que les critiques littéraires peuvent en penser ou de la manière dont ils peuvent interpréter l'œuvre ; il est conseillé aux étudiants de se laisser porter par le roman, mais d'adopter, après lecture, une distance réflexive vis-à-vis de ses propres réactions et de ce qui, dans le roman, a pu les susciter ;

B/ février 2019 : les mêmes conseils sont répétés une troisième fois ; l'auteur est présenté de façon détaillée; l'extrait (en serbe) d'une interview dans laquelle l'auteur parle de son roman est distribuée ;

$\mathrm{C} /$ un choix de descripteurs $^{6}$ du Volume complémentaire, reformulés à la première personne du singulier, est donné aux étudiants ; ils sont invités à réfléchir sur ces descripteurs et à se poser la question s'ils peuvent leur être utiles et, si oui, d'en choisir un ou deux ;

D/ 9 mars 2019 : l'activité est réalisée en cours ; temps mis à disposition : 1h 45 minutes ; utilisation du dictionnaire : autorisée ; les étudiants pouvaient se servir de citations en serbe préparées à l'avance ou choisies sur place.

Consigne :

Selon certains critiques littéraires, il nous reste du tête à tête avec Crnjanski le bonheur d'avoir rencontré un homme d'une érudition éblouissante et d'un profond humanisme. Et vous, que vous reste-t-il de la lecture du Roman de Londres ? Ecrivez un texte construit d'environ 250 mots.

E/ cours suivant (11 mars 2019) : les étudiants qui ont réalisé l'activité répondent à la question :

Да ли сте у задатку рађеном у суботу 9. марта 2019. године успели да на француском језику изразите све што сте желели у вези са својим доживљајем Романа о Лондону?

A/ Ако је одговор потврдан, објасните укратко на српском језику на који начин сте се припремили за тај рад.

Б/ Ако је одговор одречан, додајте укратко на српском језику оно што нисте успели да изразите. ${ }^{7}$

\footnotetext{
${ }^{6}$ En annexe de ce travail
} 
Quant à la recherche elle-même, elle s'est déroulée selon le scénario schématisé par Roy et Prévost $(2013,136)$, et dont nous présentons les éléments principaux :

1. planification, engagement des parties prenantes, diagnostique de l'état de situation (correspond à nos phases $\mathrm{A} /, \mathrm{B} /$ et $\mathrm{C} /$ ) ;

2. collecte de données (correspond aux phases D/ et E/ );

3. réflexion et évaluation (correspond au travail menant à l'écriture du présent article).

\section{Quelques données quantitatives}

- Nombre d'étudiants ayant réalisé l'activité : 30 sur 55 étudiants fréquentant le cours de deuxième année

- Nombre de productions retenues : 28 (2 ont été éliminées pour cause de plagiat)

- Nombre d'étudiantes : 25

- Nombre d'étudiants : 3 (dont un étranger)

- Nombre de redoublants : 2

\section{ENQUETE}

- Nombre d'étudiants ayant répondu à l'enquête : 26

- Nombre d'étudiants ayant répondu OUI : 13

- Nombre d'étudiants ayant répondu NON : 13

\section{RÉSULTATS DE LA RECHERCHE :}

\section{ANALYSE DES PRODUCTIONS PAR L'ENSEIGNANT-ÉVALUATEUR-CHERCHEUR}

Les contenus des productions des étudiants ont été analysés et évalués d'après la grille suivante :

\section{CRITERES D’ANALYSE DES PRODUCTIONS}

$\mathrm{A} /$

- Compréhension du sujet et respect de la consigne

- Compréhension de l'œuvre et mise en relation avec le sujet

- Capacité à exprimer sa pensée, originalité

- Distanciation par rapport à l'œuvre

- Correspondance du contenu avec les descripteurs choisis

- Pertinence des citations choisies $\mathrm{B} /$

- Structure de la production

- Cohésion et cohérence $\mathrm{C} /$

- Compétence morphosyntaxique

- Compétence lexicale

\footnotetext{
${ }^{7}$ Avez-vous réussi, dans le travail réalisé le samedi 9 mars, à exprimer en français tout ce que vous souhaitiez dire à propos de vos réactions au Roman de Londres ?

A/ Si oui, expliquez brièvement en serbe comment vous vous êtes préparé pour ce travail. (L'étudiant étranger a été invité à répondre dans sa langue maternelle).

$\mathrm{B} / \mathrm{Si}$ non, ajoutez brièvement en serbe ce que vous n'avez pas réussi à exprimer.
} 
Pour des raisons de place, nous ne rendrons compte ici que des résultats concernant les critères sous $\mathrm{A} /$, tandis que les critères sous $\mathrm{B} /$ et $\mathrm{C}$ / seront rapidement commentés.

Compréhension du sujet et respect de la consigne

La majorité des étudiants, 22 sur 28, a compris le sujet et produit le type d'écrit exigé, à savoir un essai sur ce que leur a apporté l'œuvre, et 26 sur 28 ont respecté la consigne de longueur de la production. Les 6 étudiants qui n'ont pas produit le type d'écrit exigé se sont contentés de résumer l'œuvre. Tous les autres ont parlé, avec plus ou moins d'habileté, des émotions que le roman a suscitées chez eux, des interrogations qu'ils se sont posées à la lecture du roman, des expériences - les leurs, ou celles de personnes qu'ils connaissent qu'ils ont pu reconnaitre.

Compréhension de l'œuvre et mise en relation avec le sujet

De manière générale, les étudiants ont montré une compréhension correcte de l'œuvre (seuls, deux étudiants semblent ne pas en avoir saisi le sens - ils ne l'ont peut-être pas lue...), bien qu'ils aient eu tendance, le plus souvent, à insister sur un seul de ses aspects / thèmes (l'amour du couple ; le courage de Nadia, la tristesse et l'obsession du suicide de Repnine ; la nostalgie de la patrie perdue ; l'incapacité d'adaptation ; la dureté de la vie des étrangers dans une métropole immense comme Londres, etc.). Ceci peut s'expliquer du fait que les contraintes de temps et du nombre de mots les ont obligés à se focaliser sur ce qui, à leur avis, était le plus important. Aucun étudiant, cependant, n'a fait, dans son devoir, le commentaire de cette nécessité de procéder à une sélection. La mise en relation avec le sujet est tout à fait satisfaisante dans 11 productions sur 28 ; on constate que seules 3 productions n'établissent pas de lien entre le sens véhiculé par l'œuvre et le sujet traité.

Capacité à exprimer sa pensée, originalité

Les productions sont très variées et le plus souvent très originales et authentiques (9 productions) ou satisfaisantes quant à la capacité des étudiants à exprimer leur pensée (8). Sur les 11 productions qui restent, on constate une forte corrélation avec le critère Compréhension du sujet et respect de la consigne : en effet, on ne peut parler d'originalité dans les devoirs qui résument le roman (4), ou ceux où le sujet n'est pas du tout traité (par exemple, le devoir dans lequel Repnine «s'explique » à la première personne ; celui où on parle de tolérance en général, du changement, etc.), ces devoirs semblant avoir été préparés à l'avance. La très grande majorité des étudiants a apprécié le roman, certains allant jusqu'à affirmer que « tout le monde devrait le lire », « je vais le proposer à tous ceux qui aiment la littérature serbe », mais il y a également des avis très critiques (« Je n'aime pas ce roman, mais $[\ldots] »)$ ou des avis critiques sur l'un de ses aspects (le personnage de Repnine « est un Russe complètement mort, sans sang ») et en même temps très positifs sur un autre («la description est incroyable », Crnjanski «nous donne tellement de couleurs et de description qu'elles nous permettent de plonger dans l'atmosphère [...]»). Les productions qui nous ont marqué le plus sont celles où sont évoquées des expériences personnelles des étudiants, dont le souvenir a été ravivé par la lecture du roman : le sentiment de nostalgie d'une étudiante qui a dû quitter le Kosovo avec sa famille, l'indigence comme cause d'éclatement d'une famille, l'amour maternel qui fait tenir bon dans les moments les plus difficiles, l'obsession du suicide d'un membre proche de la famille, etc.

Distanciation par rapport à l'œuvre

Parmi les critères dont nous nous sommes servi pour notre analyse du contenu des productions, c'est sans doute celui où nous avions le moins d'attentes, l'expérience de dix 
ans dans la lecture de ce type de devoirs nous ayant prouvé que les étudiants de deuxième année sont encore des lecteurs assez naïfs. Si la majorité se laisse porter par le roman, peu sont ceux qui se posent la question (que nous leur avions pourtant « soufflée » à plusieurs reprises), à savoir: comment l'auteur s'y prend-il pour susciter des émotions aussi violentes chez le lecteur ? Une seule production peut être considérée comme exemplaire à cet égard : l'étudiante y évoque le style poétique, le rôle des répétitions, l'emploi exagéré des virgules, etc. Quelques autres (3) sont également intéressantes de ce point de vue : les questions qu'une étudiante pose à l'auteur créent une sorte de dialogue entre la lectrice et Crnjanski ; la mise en valeur du rôle de l'atmosphère, du temps gris dans le récit comme reflet de la nostalgie ; la sélection de quelques images particulièrement poignantes, etc.

Correspondance du contenu avec les descripteurs choisis

Sur les 19 étudiants qui ont choisi d'utiliser certains des descripteurs proposés (pour la liste voir l'Annexe de ce texte), presque tous ont établi une très bonne correspondance du contenu avec le/les descripteur/s choisi/s (8) ou une correspondance tout à fait satisfaisante (7). Une étudiante n'a établi aucun lien entre ce qu'elle a écrit et le descripteur choisi, et deux ont inventé leur propre descripteur. Voici encore quelques détails à ce propos :

\section{UTILISATION DES DESCRIPTEURS PROPOSÉS}

- Sur les 13 étudiants ayant répondu OUI à l'enquête, 9 ont utilisé des descripteurs (1 étudiant a utilisé 3 descripteurs, 3 ont utilisé 2 descripteurs et 5 un seul descripteur);

- Sur les 13 étudiants ayant répondu NON à l'enquête, 10 ont utilisé des descripteurs (2 étudiants ont utilisé 3 descripteurs, 1 étudiant a utilisé 2 descripteurs et 7 un seul descripteur) ;

- Presque tous les descripteurs proposés ont été choisis, à l'exception du descripteur $\mathrm{B} 1^{8}$ Expliquer de façon détaillée avec quel personnage je m'identifie le plus et pourquoi.

- et du descripteur B2 ${ }^{9}$ Apprécier la façon dont l'ouvre favorise l'identification avec les personnages et donner des exemples;

- Les descripteurs le plus souvent choisis (par 5 étudiants) ont été les suivants :

- Décrire les émotions ressenties à certains passages du récit, par exemple au moment où j'ai craint pour un personnage, et en expliquer la raison. $\left(\mathrm{B} 1^{10}\right)$ et

- Décrire l'émotion suscitée par l'œuvre et expliquer pourquoi elle a déclenché cette réaction. $\left(\mathrm{B} 2^{11}\right)$

Pertinence des citations choisies

Seuls 9 étudiants ont choisi d'illustrer leurs propos à l'aide de citations. Les citations en question ont été correctement choisies, mais ont été, le plus souvent, utilisées pour illustrer de façon redondante, c'est-à-dire sans réelle nécessité, une phase du récit, et bien plus rarement pour étayer une affirmation de l'étudiant.

$\underline{\text { Structure de la production. Cohésion et cohérence }}$

Pour conclure cette analyse, soulignons que c'est au niveau de ces deux critères - la structure de la production et ses cohésion et cohérence - que l'on remarque la plus forte empreinte de la culture éducative, comme nous l'avons déjà mentionné plus haut. Bien que rares soient les devoirs manquant de cohésion et de cohérence, la structure en est

\footnotetext{
${ }^{8}$ Exprimer une réaction personnelle à l'égard des textes créatifs (incluant la littérature)

${ }^{9}$ Analyser et critiquer des textes créatifs (incluant la littérature)

${ }^{10}$ Exprimer une réaction personnelle à l'égard des textes créatifs (incluant la littérature)

${ }^{11}$ Exprimer une réaction personnelle à l'égard des textes créatifs (incluant la littérature)
} 
presque toujours très libre et les productions ressemblent plus à un flux de pensées, d'impressions et d'émotions, qu'à un texte structuré et argumenté. Ceci est visible dans la mise en page : sur les 28 devoirs analysés, par exemple, seuls 5 contiennent des paragraphes distincts. Sur les 23 restants, 7 placent la conclusion dans un paragraphe séparé, les autres représentent un bloc de texte unique.

\section{Compétence morphosyntaxique. Compétence lexicale}

La compétence morphosyntaxique et la compétence lexicale pourraient représenter le sujet d'un article à part entière qui traiterait de la nature des erreurs, de la persistance d'erreurs fossilisées de niveaux inférieurs à B1 et ainsi de suite. Constatons tout de même que pour les deux compétences, les étudiants ont prouvé de les posséder approximativement aux niveaux attendus en deuxième année (B1 et B2). Sur les 28 devoirs, cependant, seuls 3 ont obtenu des notes maximales pour ces deux compétences, ce qui est un résultat plutôt modeste étant donné la possibilité, pendant la réalisation de l'activité, de se servir de dictionnaires.

\section{RÉSULTATS DE LA RECHERCHE : ANALYSE DU RESSENTI DES ÉTUDIANTS}

Comme nous l'avons déjà indiqué, la deuxième partie de notre recherche porte sur les réponses des étudiants (en langue maternelle) à la question donnée dans la mini-enquête qui a suivi la rédaction du devoir.

Réponses affirmatives

Sur les 26 étudiants qui ont répondu à l'enquête, 13 ont donné une réponse affirmative : ils considèrent avoir pu exprimer ce qu'ils souhaitaient. Sur ces 13 étudiants, 6 disent avoir exprimé l'essentiel, en utilisant (pour 3 d'entre eux) un français simple, à leur portée, mais qui n'a pas permis d'exprimer toute la complexité de l'œuvre et de ce qu'elle leur a apporté. Deux étudiants considèrent que les descripteurs les ont aidés dans le travail.

Tous ces étudiants ont réalisé des activités préparatoires comme :

- s'informer sur l'auteur et la genèse du roman (2)

- essayer mentalement de faire l'analyse directement en français, sans formulation préalable en serbe ou en langue maternelle (2)

- noter ses réflexions dans un cahier (1)

- chercher dans le dictionnaire les expressions qui leur sont venues à l'esprit en serbe pendant la tentative d'analyse mentale (3)

- chercher dans le dictionnaire les mots et expressions qui apparaissent fréquemment dans le roman (1)

- lire des textes de critiques littéraires (3)

- lire l'interview de l'écrivain (2)

- noter des citations (1)

- souligner les parties du roman particulièrement importantes (1)

- écrire à la maison le brouillon du devoir ${ }^{12}(1)$

- traduire les parties du roman particulièrement importantes (1).

La comparaison des activités de préparation des trois étudiantes qui ont été le mieux évaluées par l'enseignant-évaluateur-chercheur sur la totalité de la production montre peu

\footnotetext{
${ }^{12}$ Cette stratégie est assez étonnante étant donné que les étudiants n'avaient pas connaissance de la formulation exacte de la consigne avant le samedi 9 mars.
} 
de ressemblances, ce qui prouve qu'il existe bien des stratégies préparatoires individuelles, en lien, sans doute, avec le style cognitif individuel et leurs caractéristiques et compétences (cognitives notamment) générales individuelles. Ceci remet en question la pertinence des conseils prodigués par l'enseignant-évaluateur-chercheur, conseils qui, à ce qui semble, ne peuvent être donnés qu'à titre indicatif.

\section{Réponses négatives}

Sur les 26 étudiants qui ont répondu à l'enquête, 13 ont donné une réponse négative. Contrairement au groupe d'étudiants qui ont répondu affirmativement et chez qui nous avons constaté une grande hétérogénéité de réponses et de stratégies, les réponses de ce deuxième groupe sont beaucoup plus homogènes : la très grande majorité, 10 étudiants sur 13, affirment avoir eu des difficultés de vocabulaire, mettent en avant leur incapacité à traduire de façon satisfaisante leurs pensées en français, ce qui les a poussés à les simplifier - d'où un sentiment d'insatisfaction, bien que deux de ces productions aient été jugées comme excellentes et deux, dont une qui a obtenu le maximum de point aux critères linguistiques, comme plus que satisfaisantes par l'enseignant-évaluateur-chercheur. Une étudiante affirme ne pas avoir eu suffisamment de temps, et une autre que le nombre de mots autorisés était insuffisant (ces deux productions ont été jugées comme étant les plus faibles du groupe). Une étudiante affirme ne pas avoir pu tout raconter, tout commenter, montrant ainsi qu'elle n'a pas compris la consigne, et un étudiant est mécontent du fait de n'avoir pas pu contrôler sa production, d'avoir consacré trop de place à un aspect, alors qu'il avait eu une autre idée de départ.

Ce qui caractérise la plupart des réponses de ces étudiants est une idée très élevée de ce qu'aurait dû être leur production, qui les pousse à considérer ce qu'ils ont réellement écrit comme une variante insuffisante, simpliste, appauvrie de ce qu'ils auraient pu dire dans leur langue maternelle. Ces réponses témoignent également que la stratégie mise en œuvre par ces étudiants - analyse mentale ou écrite en serbe, puis traduction - s'est montrée souvent inadaptée (mais pas toujours, comme le prouvent les deux productions très bien notées), et certainement toujours porteuse de frustration.

Seule une étudiante a exposé clairement ce qu'elle aurait voulu exprimer encore (ce qui, dans le roman, l'a poussée à réfléchir sur l'existence, quel personnage et quel destin l'ont le plus touchée).

Pour conclure ce commentaire, ajoutons que c'est dans ce groupe que nous avons repéré certains problèmes au niveau de la compétence en langue maternelle (erreurs importantes de syntaxe et d'orthographe serbes).

\section{REMARQUES CONCLUSIVES}

Notre recherche contextualisée nous a donné des réponses assez précises sur les questions que nous nous posions à propos de l'activité pédagogique présentée plus haut. Voici quelques éléments de ces réponses :

- l'activité en question est un réel défi pour les étudiants qui ont décidé de la réaliser : sa préparation leur a demandé du temps et un effort considérable, vue la longueur (deux tomes, 770 pages dans l'édition de Nolit, 1972) et la complexité du roman ; en fin de compte, presque la moitié des étudiants de deuxième année a renoncé à y participer, ce qui est bien un indice dont il faut tenir compte ;

- la difficulté de la tâche, cependant, ne semble pas excessive pour ceux qui ont réalisé l'activité, la majorité des productions ayant été évaluées comme excellentes (5), très bonnes (6) ou satisfaisantes (10) ; 7 productions ont été jugées peu satisfaisantes ; 
- nous n'avons pas pu établir de corrélation entre la qualité des productions, c'est-àdire le résultat de notre évaluation critériée, et le ressenti des étudiants ; il y a tout de même plus de productions jugées peu satisfaisantes dans le groupe d'étudiants qui ont répondu négativement à l'enquête ;

- dans le groupe des étudiants satisfaits de leur travail, la maîtrise des stratégies de planification et de préparation semble plus élevée que dans le groupe des étudiants insatisfaits, qui manifestent une frustration due à une stratégie inappropriée ;

- le nombre de garçons, de redoublants et d'étrangers étant trop faible, aucune conclusion n'a pu être tirée à leur propos.

Les recherches contextualisées sont peu propices à la généralisation. Néanmoins, celleci nous a permis d'établir les avantages et les inconvénients de l'activité que nous avons proposée à nos étudiants. Les difficultés observées, mais les réussites aussi, nous montrent que certaines recommandations peuvent être formulées :

La médiation linguistique, et en particulier les tâches qui mettent le texte littéraire au cœur de cette activité langagière, devrait, à notre avis, être comprise et travaillée dans le contexte du renforcement de la compétence interculturelle. Cette compétence repose, en effet, sur un bagage spécifique marqué profondément par la culture éducative des étudiants. Et si « la zone de construction et d'élargissement d'un répertoire linguistique et culturel pluriel» prévoit, entre autre, la mise en œuvre de « démarches d'apprentissage plus systématiques, plus contrôlées », il existe « une zone intermédiaire » avec, parmi d'autres, « une compétence de décentration» et «une compétence de distanciation» (Candelier coord. 2007, 33) qui ne vont pas de soi. Le renforcement de ces compétences est un travail de longue haleine, tout aussi important que le travail sur la compétence lexicale et morphosyntaxique, qui demande de la persévérance et une capacité d'adaptation aussi bien aux étudiants qu'à l'enseignant. Mais il porte ses fruits, comme nous avons pu le constater avec plaisir en lisant les productions de nos étudiants.

\section{ANNEXE}

Descripteurs proposé aux étudiants

TRAVAIL ECRIT SUR LE ROMAN DE LONDRES

DESCRIPTEURS

(sélectionnés et adaptés d'après Les échelles de descripteurs $d u$ CECR)

A/ EXPRIMER UNE REACTION PERSONNELLE A L'EGARD DES TEXTES CREATIFS (INCLUANT LA LITTERATURE)

JE PEUX :

1. Décrire les émotions ressenties à certains passages du récit, par exemple au moment où j'ai craint pour un personnage, et en expliquer la raison.

2. Relier les émotions ressenties par un personnage à celles que j'ai vécues.

3. Associer les événements du récit avec des événements similaires que j’ai vécus ou dont j'ai entendu parler.

4. Expliquer de façon détaillée avec quel personnage je m'identifie le plus et pourquoi.

5. Expliquer pourquoi certaines parties ou certains aspects de l'œuvre m'ont particulièrement intéressé/e.

6. Exprimer de façon détaillée mes réactions à la forme d'expression, au style et au contenu de l'œuvre et expliquer ce que j'ai apprécié et pourquoi. 
7. Décrire l'émotion suscitée par l'œuvre et expliquer pourquoi elle a déclenché cette réaction.

8. Présenter clairement mes réactions vis-à-vis de l'œuvre, développer mes idées et les étayer par des exemples et des arguments.

B/ ANALYSER ET CRITIQUER DES TEXTES CREATIFS (INCLUANT LA LITTERATURE)

JE PEUX :

1. Décrire les thèmes et les personnages clés du récit.

2. Désigner les épisodes et les événements les plus importants du récit et en expliquer la signification ainsi que les liens.

Apprécier la façon dont l'œuvre favorise l'identification avec les personnages et donner des exemples.

\section{RÉFÉRENCES BIBLIOGRAPHIQUES}

Beacco, Claude. «Les cultures éducatives et le Cadre européen commun de référence pour les langues ». Revue japonaise de didactique du français. Vol. 3, n. 1, 2008, Etudes didactiques.

Candelier, Michel, coord. A travers les langues et les cultures. Graz, Centre européen pour les langues vivantes, 2007.

CECR. Cadre européen commun de référence pour les langues : apprendre, enseigner, évaluer. Paris, Les Editions Didier, 2001.

Đurić, Ljiljana. «Traduction et/ou médiation: quelles perspectives pour une évolution possible de l'enseignement universitaire du français en Serbie ? », dans Les Etudes françaises aujourd'hui, Tradition et modernité. Niš, Filozofski fakultet, Izdavački centar Univerziteta u Nišu, 2016, p. 437-448.

Pešić, Mirjana, et al. Pedagogija u akciji. Beograd, Institut za pedagogiju i andragogiju i Filozofski fakultet Univerziteta u Beogradu, 1998.

Puren, Christian. «Explication de textes et perspective actionnelle : la littérature entre le dire scolaire et le faire social », 2006. http://www.aplv-languesmodernes.org/

Puren, Christian. «Perspectives actionnelles sur la littérature dans l'enseignement scolaire et universitaire des langues-cultures: des tâches scolaires sur les textes aux actions sociales par les textes », 2012. http://www.christianpuren.com

Roy, Mario, et Paul Prévost. «La recherche-action : origines, caractéristiques et implications de son utilisation dans les sciences de la gestion », Recherches qualitatives - vol. 32(2), 2013, p. 129-151. http://www.recherche-qualitative.qc.ca/revue.html

Srebro, Milivoj. CRNJANSKI / TSERNIANSKI /, MILOŠ (1893 - 1977). Serbica, revue électronique. http://serbica.ubordeaux-montaigne.fr

Volume complémentaire avec de nouveaux descripteurs. Strasbourg, Conseil de l'Europe, 2018.

Weissmann, Dirk. «La médiation linguistique à l'université : propositions pour un changement d'approche. » $E L A \mathrm{n}^{\circ} 167,2012$, p. 313-324.

\section{KNJIŽEVNO DELO U SREDIŠTU JEZIČKE MEDIJACIJE NA UNIVERZITETSKOM NIVOU: UKRŠTANJE VIĐENJA}

Zajednički evropski okvir za žive jezike (2001) uveo je pojam jezičke medijacije, dajući snažan podsticaj za promenu tradicionalne aktivnosti prevođenja na času stranog jezika (koji, na univerzitetskom nivou, uglavnom ima oblik prevođenja književnih tekstova), promena koja je išla u pravcu uvođenja raznih oblika preformulisanja uglavnom neknjiževnijh tekstova. Nudeći nove skale deskriptora - Izražavanje lične reakcije na kreativne tekstove i Analiza i kritika kreativnih tekstova Dopunska knjiga deskriptora, koju je Savet Evrope objavio 2018, stavlja književni tekst u središte jezičke 
aktivnosti medijacije. Ovaj članak predstavlja rezultate kontekstualizovanog interpretativnog istraživanja koje ima za cilj da proveri, polazeći od pismene jezičke aktivnosti medijacije (sa srpskog na francuski jezik) u kontekstu univerzitetske nastave, da li jezička kompetencija studenata druge godine koji studiraju francuski jezik $i$ književnost, a za koju je procenjeno da odgovara približno nivoima B1 i B2, omogućuje da analiziraju, vrednuju i tumače na francuskom jeziku složeno književno delo kao što je Roman o Londonu Miloša Crnjanskog. Kvalitet studentskih radova dovodi se u vezu sa ponuđenim deskriptorima s jedne strane $i$, s druge strane, sa stepenom zadovoljstva sopstevnim radom samih studenata. Daju se obaveštenja i o prirodi primenjenih strategija planiranja i njihovom učinku.

Ključne reči: univerzitetska nastava francuskog jezika u Srbiji, pisana medijacija L1-L2,

književni tekst, lična reakcija, strategije planiranja, obrazovna kultura 